\title{
Le projet didactique de Fénelon auteur de Télémaque : enjeux et perspectives
}

Henk Hillenaar

\section{(2) OpenEdition Journals}

Édition électronique

URL : https://journals.openedition.org/dhfles/1478

DOI : $10.4000 /$ dhfles. 1478

ISSN : 2221-4038

Éditeur

Société Internationale pour l'Histoire du Français Langue Étrangère ou Seconde

Édition imprimée

Date de publication : 1 juin 2003

Pagination : 11-23

ISSN : 0992-7654

Référence électronique

Henk Hillenaar, «Le projet didactique de Fénelon auteur de Télémaque : enjeux et perspectives »,

Documents pour l'histoire du français langue étrangère ou seconde [En ligne], 30 | 2003, mis en ligne le 01 janvier 2012, consulté le 27 mai 2021. URL : http://journals.openedition.org/dhfles/1478 ; DOI : https://doi.org/10.4000/dhfles.1478

Ce document a été généré automatiquement le 27 mai 2021

(c) SIHFLES 


\title{
Le projet didactique de Fénelon auteur de Télémaque : enjeux et perspectives
}

\author{
Henk Hillenaar
}

1 Mon sujet se présente comme un dyptique : il s'agira d'abord des enjeux de Télémaque, puis des perspectives que peut ouvrir ce livre si célèbre. Par enjeux j'entends l'intérêt que Les Aventures de Télémaque ont représenté aux yeux de son auteur, l'intérêt aussi que lui a accordé le public de son temps. Les perspectives, par contre, sont celles que nous-mêmes, lecteurs d'aujourd'hui, pouvons adopter en étudiant cet ouvrage. Télémaque qui pendant longtemps fut le livre le plus lu, ou du moins le plus réédité et traduit de la littérature française, connaît depuis une dizaine d'années, et après une éclipse qui dura longtemps aussi, un regain de faveur remarquable. Notre colloque ici n'est que l'une des preuves de ce retour en grâce d'un ouvrage qui se révèle être une mine pour tous ceux qui, littéraires, philosophes, théologiens ou pédagogues, veulent enrichir leur savoir au sujet du grand siècle français, ou, plus simplement, goûter la saveur, ce « je ne sais quoi de pur et de sublime », de ce grand livre (cf. Lanavère 1994).

\section{Enjeux}

\section{Le miroir des princes}

2 Télémaque s'insère dans la longue série d'ouvrages consacrés à l'éducation du prince que la France et l'Europe ont connus depuis le Moyen Age (voir Halévi 2001). Leur importance a été telle qu'on s'est assez vite mis à les considérer comme un 'genre littéraire' à part, appelé 'Miroir des Princes'. Il s'agit d'ouvrages parlant tantôt des devoirs du souverain, tantôt de la formation politique du jeune prince, ou encore de l'éducation princière en général. De la masse de ces 'Miroirs' enterrés dans nos bibliothèques émergent celui de Thomas d'Aquin, De Regimine Principis (1265), et, des siècles plus tard, ceux d'Érasme, Institutio Principis Christiani (1516) et de Guillaume 
Budé, Institution d'un Prince Chrétien (1547). Volker Kapp qui a étudié Les aventures de Télémaque dans ce contexte, a retrouvé, rien que dans la France du XVIIe siècle, plus de cent cinquante 'Miroirs des Princes' (Kapp 1982 : 209-220). On comprend facilement l'intérêt suscité par de tels ouvrages. Destinés à l'éducation princière, ils devenaient exemplaires de toute éducation et servaient de modèle et de référence pour le public cultivé de l'époque. Du reste, les auteurs de Miroirs avaient l'habitude, en publiant leurs traités, de s'adresser non pas seulement au personnage princier mais aussi à un public plus large. Ces livres figuraient aux côtés des traités de pédagogie 'normaux', dont les plus importants à l'époque étaient Le traité des études de Claude Fleury (1686), qui devint sous-précepteur à Versailles, les Règles de l'éducation des enfants (1687) de Pierre Coustel et, bien entendu, le Traité de l'éducation des filles (1687), de Fénelon lui-même.

Fénelon a certainement bien connu les études de ses contemporains, surtout celles de son collègue Claude Fleury. Le parallèle entre les 'mœurs des Israélites' décrites par ce dernier et les mœurs des civilisations dont parle le Télémaque a été maintes fois étudié. Nous pouvons également supposer qu'il avait lu Érasme et Budé, toujours très présents vers 1700 . Cependant, les ouvrages dont il s'est le plus servi au moment d'arriver au poste de précepteur des princes, étaient les 'miroirs des princes' composés par Bossuet, qui était alors non seulement son prédécesseur mais aussi son ami. Le matériel, manuscrit, élaboré par Bossuet et son entourage pour l'éducation du Grand Dauphin pour la génération précédente donc - fut réutilisé par le nouveau précepteur : catéchisme, grammaire, traité de mathématiques, cours d'histoire, papiers d'où sortiraient par la suite notamment la Politique tirée de l'Écriture sainte, et le Discours sur l'histoire universelle (cf. Le Brun 2001). Tout cela nous renseigne sur le contenu de l'enseignement de Bossuet, et jusqu'à un certain point sur celui de Fénelon aussi. Quant à la forme qu'adopta ce même enseignement, nous sommes beaucoup mieux renseignés sur la méthode de Fénelon que sur celle de Bossuet. Les textes littéraires du premier, ses Fables, Dialogues des morts et Télémaque, bien sûr, nous font voir comment Fénelon a dû opérer. Ce qui comptait d'abord dans son enseignement, c'était la relation entre maître et disciple. Il chérissait le dialogue socratique et la sentence, facile à mémoriser. Il racontait des fables que l'élève n'oublierait plus, et il lui apprenait à faire des discours en bonne et due forme, genre dont le Télémaque abonde aussi.

\section{Formation du sujet et oubli du moi}

4 Lisant ces ouvrages pédagogiques de l'archevêque de Cambrai, nous assistons en fait à un véritable changement de perspective. Là où pour Bossuet le principal enjeu était encore de fournir un enseignement aussi riche et complet que possible, nous voyons chez Fénelon l'accent se déplacer et aller vers l'élève. C'est lui qui est désormais le centre d'intérêt de l'éducation, et non pas la matière à enseigner. L'enjeu du Télémaque consiste moins dans la formation intellectuelle de l'élève par une série de leçons d'histoire, de politique et de morale que dans la transformation de ce même élève en un 'prince philosophe et vertueux'. Le clivage entre Bossuet et Fénelon en matière d'éducation reflète celui, plus philosophique, où tout un siècle, suivant en cela le modèle cartésien, est en train de se tourner vers le sujet humain, le Je pensant devenant le centre de l'univers philosophique, et le 'moi humain' celui de l'univers psychologique et pédagogique concomitant. Ce 'moi' - ses désirs, son amour propre, sa conscience de soi - est devenu le centre de la pensée et de la spiritualité et de la pédagogie de l'archevêque de Cambrai. En cela il est, beaucoup plus que Bossuet, le frère de Racine et 
de Madame de La Fayette. Télémaque, vaillant mais triste fils d'Ulysse, évolue dans le même univers psychologique où vivent aussi la Princesse de Clèves ou Britannicus. L'important dans tout cela est que, derrière le duo Télémaque et Mentor - ou leurs remplaçants dans le livre - nous sentions la présence de Fénelon et de son disciple. Télémaque devient ainsi, selon un mot de Jacques Le Brun, une véritable 'mise en scène de la relation pédagogique' (ibid. : 235). Ce faisant, le précepteur opère une espèce de révolution par rapport à ses prédécesseurs et prépare à sa façon le mouvement pédagogique du XVIII ${ }^{\mathrm{e}}$ siècle, Rousseau surtout, fervent admirateur de son œuvre, lui qui disait qu'il aurait voulu être le valet de chambre de l'archevêque de Cambrai.

5 Le grand enjeu de l'éducateur qui écrit Télémaque est d'inculquer à son élève l'idéal de désintéressement dans tous les domaines de la vie. «Le but unique et essentiel est de ne vouloir jamais l'autorité et la grandeur pour soi » (Fénelon 1997 : 312). Cette sentence, qui résume à la fin du livre toutes les leçons du précepteur des princes, a pour condition, cent fois répétée, elle aussi, que le futur roi remplace sa propre volonté par celle de son maître. Mentor représente 'l'ordre' auquel il doit se soumettre : ordre familial, ordre politique, ordre divin, qui exigent tous de lui - du sujet humain - l'oubli de son propre intérêt, de sa propre gloire, de ses ambitions personnelles. Les vraies valeurs se découvrent au-delà de cette soumission, de l'oubli de soi, là où, aux yeux de Fénelon, l'homme recouvre sa 'simplicité', son véritable 'naturel'.

\section{Le plaisir dans l'éducation}

6 Le contenu très austère de Télémaque n'a sans doute pas été saisi par le grand public. Ce qui, par contre, a toujours été un objet d'attraction pour ce même public est la forme du livre, autre enjeu de l'auteur, forme très agréable - certains disaient 'trop agréable' - de la 'fable antique'. Normalement, le genre du Miroir des princes, didactique et sérieux, gardait le caractère abstrait du traité politique ou philosophique (Hillenaar 1994 : 7 sq.). L'originalité de Fénelon était justement d'avoir inséré dans Télémaque une part d'agrément et de rêve. Il mettait ainsi en pratique le programme pédagogique qu'il avait présenté quelques années auparavant dans son traité De l'éducation des filles. Le public y avait lu que, pour être efficace, l'éducation des enfants doit d'abord être attrayante. Le même ouvrage préconisait en outre une éducation fondée sur ce que de nos jours nous appellerions des "leçons de choses ». C'est bien de tout cela qu'il est question dans Les aventures de Télémaque, où l'imagination humaine, malgré son caractère d'idole et de puissance trompeuse, a souvent le beau rôle. Mais le plaisir n'est qu'un moyen, une aide provisoire pour subvenir à la faiblesse naturelle de l'enfant, à celle surtout de sa raison. Pour Fénelon comme pour ses contemporains, l'éducation est en première et en dernière instance celle de la raison, c'est-à-dire de l'entendement et de la volonté de l'enfant. Jeune prêtre encore, il avait déjà écrit dans ses Dialogues sur l'éloquence: «Si quelque chose peut faciliter la vertu, c'est de la mettre d'accord avec le plaisir ; au contraire, quand on les sépare, on tente violemment les hommes d'abandonner la vertu » (Fénelon 1983 : 15).

7 On comprend donc que, dans le cas du jeune dauphin, Fénelon ait remplacé le traité rédigé dans une prose austère par la prose poétique - mi-roman, mi-épopée - des Aventures de Télémaque. "Je n'ai jamais songé qu'à amuser Monsieur le Duc de Bourgogne par ses aventures, écrit-il, et qu'à l'instruire en l'amusant » (Fénelon 1850 : VI, 666). Cela a étonné beaucoup de ses contemporains, Bossuet le premier, qui se sont 
montrés scandalisés qu'un prêtre puisse se consacrer à l'écriture d'un roman. Leurs reproches reflètent le climat culturel de l'époque et ce que l'on a pu appeler 'l'antihumanisme' de ces dernières décennies du dix-septième siècle. Le cartésianisme régnant condamnait la mémoire et l'imagination, complices de l'optimisme 'humaniste' des générations précédentes, comme des idoles dangereuses. En tant que philosophe, Fénelon n'était certainement pas insensible à ces tendances-là, lui dont les sympathies pour le cartésianisme sont bien connues. La raison et sa clarté sont au centre de son enseignement, surtout la clarté de la volonté, raison pratique qui devient l'enjeu même de l'éducation prodiguée à Télémaque ; mais il n'ira jamais aussi loin que les cartésiens, qu'un Malebranche notamment, contre qui il écrit tout un traité, parce que ce philosophe "tourne tout en raison ", oubliant que le mystère de l'homme, qui est le mystère de Dieu, ne pourra jamais être pénétré par la seule raison humaine.

La pédagogie de Fénelon ${ }^{1}$ nous montre, s'il en était besoin encore, qu'avec lui, nous avons affaire à un spirituel plus qu'à un philosophe. Le philosophe en Fénelon a beau vouloir « insinuer la raison " partout et amener le duc de Bourgogne à une meilleure connaissance de lui-même, en même temps le spirituel en lui ne cesse de prêcher, très paradoxalement, très spirituellement, que cette connaissance de soi, tant prônée par les Anciens, doit aboutir à l'oubli de ce même moi. L'humilité qui sied à la raison explique sans doute aussi que Fénelon n'hésite pas à rendre à la mémoire et à l'imagination, et au plaisir qu'elles procurent, la place que nous connaissons. Cette humilité prend chez lui la forme de la 'simplicité', et de la 'peinture naturelle' dont il voudrait se faire le champion et où la raison, au lieu d'être pure, se revêt des formes et des couleurs du monde des sens.

9 Un tel choix rejoint d'ailleurs la préférence dont fait preuve à ce moment-là, en chérissant contes, fables ou roman historiques, plus d'un esprit réagissant contre le cartésianisme ambiant, trop austère. Dans ce contexte il faut rappeler également l'amitié qui liait l'archevêque de Cambrai et son élève avec La Fontaine, les fables de ce dernier étant l'un des grands modèles de l'éducation princière. Dans l'ensemble cependant l'écriture souvent prolixe et le style presque luxuriant des Aventures de Télémaque semblent être plus proches de ceux de l'Astrée et des romans de la première moitié du siècle que de ceux de ses contemporains. S'il y a anti-humanisme dans ce roman, cela ne regarde sûrement pas la forme. Nous aurons à y revenir.

\section{Un discours chrétien}

10 L'une des performances que le précepteur des princes a accomplie en écrivant son Télémaque est certainement d'y avoir mêlé intimement, et presque imperceptiblement fable antique et fable 'mystique', discours littéraire et discours spirituel. C'était là sans aucun doute, pédagogiquement parlant, l'enjeu le plus original de son entreprise : donner une éducation chrétienne au prince de Versailles à travers le récit de l'éducation du fils du grand Ulysse. Télémaque, prince chrétien, cela peut sonner contradictoire au premier abord, mais ce tour de force, Fénelon le réussit parfaitement. Si, comme on l'a souvent fait remarquer (cf. Hepp 1968 et Lanavère 1995), Virgile avait déjà dans une certaine mesure, 'spiritualisé' la tradition homérique ; ici, chez Fénelon, nous assistons à un véritable ajustement des deux mondes, gréco-romain et chrétien, le premier devenant comme la figuration de l'autre. Et plus qu'une figuration, car l'idée d'un certain paganisme vertueux et attirant, qui annonçait et préparait le Christianisme, avait été populaire en Europe depuis le Moyen Age. À partir du milieu du 
XVII ${ }^{e}$ siècle, et sous l'influence de la Réforme, l'augustinisme et le cartésianisme régnants avaient de plus en plus relégué ce monde d'idées parmi les rêves d'un Christianisme d'antan. Fénelon y a néanmoins recours, mais en introduisant un double changement : d'abord en ne faisant plus de l'antiquité aimable une préfiguration des choses à venir, mais en représentant à travers elle réellement ses mystères. Chez lui, l'antiquité devient la métaphore du Christianisme. La doctrine de la grâce - dont Mentor peut être regardé comme la personnification - s'y voit à toutes les pages, et aussi celle du péché, de la souffrance rédemptrice et de la compassion envers ceux qui souffrent. Le relevé des passages de Télémaque empruntés plus ou moins directement à la Bible est impressionnant (Dupriez 1961 : 201-209). Cela lui est possible grâce à un autre changement de perspective : ce que nous lisons dans Les aventures de Télémaque comme doctrine chrétienne, ce n'est pas le dogme de l'Église, qui reste seulement le filigrane du texte, mais la spiritualité de Fénelon, celle du désintéressement. Les leçons de Télémaque - "Le but unique et essentiel est de ne vouloir jamais l'autorité et la grandeur pour soi » (Fénelon 1997 : 312) - ne diffèrent pas substantiellement de celles du pur amour dans Explication des maximes des saints, l'abrégé de sa spiritualité. Mentor harcèle le jeune homme de tellement d'épreuves et d'exhortations à acquérir cette attitude que Télémaque, désespéré, finit par lancer un cri que nous comprendrions difficilement sans le contexte mystique du texte : " $\hat{O}$ mon vrai père, ô Mentor, délivrez-moi de tant de maux ! Je ne puis ni vous abandonner ni vous suivre. Délivrezmoi de tant de maux, délivrez-moi de moi-même : donnez-moi la mort » (Fénelon 1997 :90).

11 Contrairement à ce qu'on a tendance à penser, dans cette merveilleuse imitation d'Homère, l'esprit d'Homère, libre, léger, fait souvent défaut. De l'Odyssée il y a surtout les belles apparences, celles-ci étant désormais investies par l'esprit d'une spiritualité austère. Si Fénelon insiste, dans ses Dialogues sur l'éloquence, sur la distance qui sépare les auteurs bibliques d'Homère - « Jamais Homère même n'a approché de la sublimité de Moïse dans ses cantiques »; " Jamais Homère [...] n'a égalé Isaïe peignant la majesté de Dieu " - le revers de cette médaille est que le Christianisme fait perdre à Homère la simplicité où hommes et dieux sont transparents, et le bien et le mal faciles à distinguer. Même si Fénelon essaie de traiter ses personnages comme son collègue Claude Fleury traitait Israélites et premiers Chrétiens, avec toutes les idéalisations et simplifications que cela suppose, son idéal propre, celui du désintéressement en toutes choses, ne lui permet pas de rester véritablement 'simple', comme le montre la citation ci-dessus. Ce sont les prouesses stylistiques de l'auteur - les apparences donc - qui réussissent, souvent aujourd'hui encore, à cacher la hardiesse et la profondeur des leçons du livre.

L'enjeu principal de Fénelon est là, à n'en pas douter, dans cette leçon de spiritualité, englobant et résumant les autres enjeux que nous venons de voir : miroir des princes, éducation 'subjective' plus qu'objective', et plaisir que l'imitation d'Homère doit procurer au jeune élève. La plupart des lecteurs contemporains n'ont pas réalisé la portée d'un ouvrage où projet pédagogique et discours spirituel étaient si intimement mêlés, ils voyaient surtout le scandale que Télémaque avait suscité dans le royaume, la critique du règne de Louis XIV que l'on se plaisait à y reconnaître. En outre le livre parut une semaine seulement après la condamnation de son auteur par Rome pour ses idées mystiques. Tout cela ne contribua pas peu à la réputation de Fénelon et de sa pédagogie, dont Télémaque deviendrait à la fois le résumé, le joyau et le symbole. 


\section{Perspectives}

13 J'entame ici le deuxième volet de mon diptyque qui concerne davantage notre regard sur Télémaque aujourd'hui : les multiples points de vue qui sont devenus possibles à nous lecteurs modernes, grâce à la distance que l'histoire - l'histoire des faits, l'histoire des idées - nous a donnée. J'en choisis trois, ouvrant ainsi trois perspectives sur le grand livre de Fénelon, trois éclairages différents : celui, d'abord, de l'histoire de la spiritualité et de la pédagogie. Puis je ferai une incursion du côté de la psychanalyse, d'une science moderne donc, essayant de tracer certaines dimensions inconscientes du livre de Fénelon. Enfin, mon troisième point de vue qui englobe en quelque sorte les précédents, concerne ce que l'on pourrait appeler 'l'anthropologie' de l'auteur de Télémaque, l'idée que celui-ci se faisait de l'homme, de ses désirs, de ses vérités, de ses lois.

\section{Histoire de la spiritualité et de la pédagogie}

Je l'ai déjà signalé : en tant qu'ouvrage pédagogique, Télémaque constitue à sa façon une illustration du mouvement d'intériorisation qui caractérise ce XVII ${ }^{\mathrm{e}}$ siècle, où le sujet humain occupe de plus en plus de place. Ce 'Miroir du prince' parle de tous les aspects de la vie : famille et mariage, nation et politique, agriculture, économie et commerce, guerre et paix, tout ce qu'on pourrait appeler la 'vie extérieure' du futur roi y a sa place, et pourtant, nous le savons dès le départ, ce qui compte aux yeux de l'éducateur, c'est le monde invisible, dont 'le moi' seul possède le secret et que Fénelon n'hésite pas à rendre visible tout au long de son livre, par toutes sortes d'artifices, de visions, de miracles même. Le livre XIV - la descente de Télémaque aux enfers - et le dernier livre où nous assistons à l'épiphanie de Mentor-Minerve, sont à cet égard les plus révélateurs.

La vérité, chez Fénelon, vient de l'intérieur. Le monde sensible, extérieur, s'il veut acquérir vérité et valeur, ne saurait le trouver qu'à partir de cet univers-là où règne la lumière de l'unité, de la simplicité, de l'harmonie. En nous donnant ses descriptions de paysages, ou ses rêves d'une civilisation idéale, Fénelon opère sans cesse ce mouvement de l'intérieur vers l'extérieur, du haut vers le bas, de l'un vers le multiple, et cela de façon presque naturelle. Le lecteur a l'impression que le monde sensible évoqué par Fénelon n'illustre pas seulement une idée mais participe directement de l'idéal, d'un monde d'en haut, divin. Les sympathies de Fénelon, nous le savons, vont toutes vers Platon, et non pas vers Aristote. Mais nous savons aussi les problèmes qu'un tel choix pose, et plus d'un commentateur a constaté que, justement, le monde sensible a chez lui peu de consistance. Si tout est participation, illustration, voie d'accès au divin, est-il encore possible d'accorder au monde sensible - à la famille, à la politique, au travail une vérité et une valeur propres? Du point de vue de la pédagogie, ce monde familial, politique, économique est l'objet d'une réforme collective, certes, mais plus encore l'occasion d'un renoncement personnel. Le dedans, au lieu d'être le support ou la vérité du monde de dehors, invite surtout à déprécier ce dernier, et à le faire oublier.

Ce que le précepteur demande principalement au prince, c'est une attitude d'indifférence. Le 'moi' de l'élève, centre de ses désirs doit s'effacer et disparaître, entraînant dans sa chute tout ce qui 'l'intéresse', tout ce qui fait l'étoffe de la vie 
quotidienne, concrète. Désinvesti par un 'moi' indifférent, tout cela n'a plus de poids ou de consistance. Le lecteur, lui, avec les acquis de la pédagogie moderne en tête, se demande ce que représentent alors le mariage à venir de Télémaque et d'Antiope, la prospérité retrouvée de Salente, et la paix entre les peuples de la Méditerranée. Le visible n'existe qu'en fonction de l'invisible, du divin, ou, comme Fénelon aime l'exprimer, de l'Un, de l'unité divine. Sa réalité est une réalité de participation, qui manque d'assise, de base propre. Télémaque participe déjà de la félicité des bienheureux. Or celle-ci, nous dit Fénelon, « vient du dedans, et ne leur laisse aucun sentiment pour tout ce qu'ils voient de délicieux au dehors » (Fénelon 1997 : 247).

Dans une remarquable étude du style de Fénelon - l'un des meilleurs articles que je connaisse - Françoise Berlan (1986 et 1995) fait de l'archevêque de Cambrai « un poète de l'immatériel ». Elle constate que chez lui « le réel n'est pas dans les objets mais dans une révélation qui est un verbe. Le vrai est accordé de l'extérieur par le divin, il est luimême de l'ordre de la représentation sensorielle, affective, spirituelle. Les visions, les songes, la parole de Minerve, valent pour l'expérience et la supplantent » (ibid. : 20). Dans le Télémaque, observe-t-elle très finement, « le concret et l'abstrait paraissent aller l'un vers l'autre dans une indistinction, comme si les lieux et les choses étaient d'emblée intériorisés, comme si, en échange, pensées et sentiments avaient la couleur des images " (ibid. : 11). La raison de cela, elle la trouve dans " une sensibilité qu'un excès de présence de soi-même affecte dans son rapport au monde ". Cette sensibilité s'exprime avant tout dans " une rêverie de l'air ", de l'apesanteur et de la vitesse, et Françoise Berlan d'insister sur la fréquence de verbes tels que 'fendre' et 'fondre', 'courir' et 'voler', 'lever', 'élever' 'monter', 'couronner', 'se jeter', 'se précipiter', toute une " esthétique de la rapidité aérienne ", dans laquelle elle reconnaît la " revanche de l'action » - et de la volonté - sur le manque de poids et d'assise constaté auparavant. Et elle conclut : "À côté de l'inconstance des représentations, il existe une compensation de la volonté » (ibid. : 22), qui, elle, tend, non vers l'élévation ou l'exaltation, mais vers l'ascèse et l'amortissement.

Cette analyse rejoint celle, tout aussi remarquable, de Benedetta Papasogli qui, étudiant l'espace intérieur tel que Fénelon le décrit, constate chez ce dernier " une sorte de conversion de l'image de la hauteur dans celle de la profondeur, la cime de l'âme devenant le 'fond de l'âme' ou le 'fond du cœur', mot particulièrement cher à l'archevêque de Cambrai, « le grand enjeu de [sa] spiritualité » (2000). Elle insiste sur le fait qu'en parlant de l'union avec Dieu, Fénelon a moins recours à des métaphores telle que 'montée', 'cime' 'ou 'pointe', chères à d'autres, mais qu'il préfère celles d"abîme', et surtout donc de 'fond', « état très simple de la pensée et de la volonté de l'homme [qui] débouche sur l'abîme de Dieu " (ibid. : 69). Ce même mouvement se retrouve en filigrane dans le Télémaque. Très significativement, le point culminant du livre est la descente du prince aux enfers, vers le fond de la terre. Là, la vérité se montrera à lui dans le spectacle réel/irréel du sort réservé aux mauvais rois puis aux bons rois. L'idéal du précepteur s'y révèle être, définitivement, l'obéissance à une volonté supérieure, celle du fils qui suit le père. Nous sommes encore à mille lieues de la pédagogie moderne. Celle-ci essaie de former la volonté propre de l'enfant, chez Fénelon il s'agit de suivre la volonté de plus grand que soi. Benedetta Papasogli interprète ce phénomène dans la perspective christologique du Fils qui s'offre en victime au Père. Mais la plupart des commentateurs s'accordent pour dire qu'il n'y a pas de christologie plus faible que celle de l'archevêque de Cambrai. Celui-ci parle beaucoup de Dieu, et très peu de Jésus-Christ. Il paraît plus indiqué de regarder d'abord du côté des fils et des 
pères qui peuplent le Télémaque car ce livre abonde en fils qui cherchent un père, et chose plus sérieuse, en fils qui sont tués par leur père.

\section{Psychanalyse}

19 Cela nous invite à jeter un regard du côté de la psychanalyse, c'est-à-dire à voir d'abord comment Fénelon traite les deux instances qui sont décisives pour le développement d'un être humain : l'instance maternelle et l'instance paternelle. Je me permets ici de résumer très succinctement l'essentiel de ce que j'ai avancé ailleurs (Hillenaar 1994). Car si Fénelon met en scène la recherche du père, si Télémaque a tant besoin de la protection et de la sagesse d'un père - fortement idéalisé, représenté par de multiples vieillards, et surtout par Mentor - c'est qu'une menace pèse sur le jeune prince, du début jusqu'à la fin du livre, c'est la menace de la mère et de la femme. Non seulement de Calypso, la belle séductrice, mais de presque toutes les autres femmes qui dans le livre n'ont jamais le beau rôle. Pénélope, sa mère, l'une des figures les plus attachantes de l'Odyssée, Fénelon la transforme en un être dangereux, une nouvelle Clytemnestre même ; la même chose est vraie dans le cas de Calypso et de presque toutes les femmes du livre, excepté, bien sûr, d'Antiope, créée à l'image de la femme forte du Livre de la Sagesse. Fénelon s'attarde longuement à décrire la cruauté de la femme - Calypso, Astarbé - très peu leur beauté. La grande peur de l'auteur de Télémaque est que ses héros aient une apparence 'efféminée', cette menace revenant comme une obsession dans le livre. Il n'empêche que la beauté est l'apanage des jeunes guerriers qui vont mourir, et que Fénelon décrit avec un lyrisme qui ailleurs est rare chez lui. Le désir homo-érotique semble ainsi remplacer celui de la mère et de la femme.

Bien entendu, on ne saurait exister sans mère, et la nostalgie du monde du commencement, le monde de la bonne mère, est omniprésent dans le livre, mais toujours de façon indirecte : dans l'amour de l'auteur pour le monde de la nature et des sens, dans son choix du cadre mythologique - pensez à la fameuse scène d'Amphitrite surgissant des eaux, portant son nourrisson Palémon dans son sein - dans son évocation d'un âge d'or, dans la prose poétique qu'il adopte et dans son idéal d'une symbiose avec les dieux, et avec le père. Les pères du Télémaque, Mentor le premier, ont souvent aussi quelque chose de maternel. Aussi savons-nous que derrière Mentor se cache Minerve. Si le livre de Fénelon a eu un succès si immense, c'est d'abord à cause de ce climat de douceur et de beauté dans lequel le texte baigne. Ce décor permet de faire passer le message spirituel du précepteur. Du reste, dans cet univers où le personnage de la mère est toujours décevant, la figure du père n'offre que peu de compensation au jeune prince. Le père est lointain, trop haut, presque inaccessible et, ce qui est essentiel, réduit, en dernière instance, à être volonté pure, séparée de la mère et de tout ce que celleci représente : la terre, le corps, le plaisir. Qui pis est, le père se révèle être celui qui tue. Télémaque est le livre où les pères tuent et où les fils meurent. Ce livre qui aspire à 'tuer le moi' baigne souvent dans un climat que de nos jours on appellerait sans doute 'sado-masochiste'. L'histoire de Salente, Fénelon la fait commencer par celle du fils d'Idoménée tué par son propre père. Cette première victime est suivie de nombreux autres jeunes gens qui meurent. La voix qui inspire celle de Mentor se révèle être celle de Minos, le dieu des enfers et de la mort. Or, plus encore que des leçons de modération et de partage, c'est-à-dire des leçons de vie, Minos et Mentor enseignent des leçons de désert, de renoncement, de mort. Paradoxalement, avec le Platonisme de 
Fénelon, le Christianisme semble perdre de vue son premier enjeu : incarnation et rédemption semblent absentes ici, sans représentation.

\section{Anthropologie}

21 J'adopte donc, pour terminer, une troisième perspective, plus générale, que j'appelle 'anthropologique', me demandant, avec la distance que nous avons désormais, quelle idée l'auteur de Télémaque se faisait de l'homme, c'est-à-dire quelle est son attitude à l'égard des deux phénomènes qui, à nos yeux post-modernes, semblent le définir : le désir et la loi. Chez Fénelon, la loi est partout, le désir, par contre, qui est fatalement désir du moi, et désir de plaisir, est surtout l'objet de sa méfiance et de son refus. Il n'est reconnu et accepté qu'indirectement, nostalgiquement, dans le cadre tout en douceur et en beauté qui est l'une des raisons de la célébrité de l'ouvrage, plaisir qui doit avoir pour effet l'obéissance à la loi. Ainsi dans Télémaque nous nous heurtons à un conflit entre le programme collectif, positif surtout, et le projet personnel qui, lui, est surtout négatif. C'est ce programme heureux que les Lumières ont admiré et adopté : justice, tolérance, bonheur des peuples. On n'a sans doute pas assez vu que ce beau programme avait été rendu possible, en dernière analyse, par un acte de désintéressement incompréhensible : par le meurtre du fils d'Idoménée par son propre père. Dans cette vision des choses - reprise littéraire des idées mystiques de l'Explication des maximes des saints - Fénelon partage le pessimisme et la haine contre le 'moi' et ses désirs de beaucoup de ses contemporains, tels La Rochefoucauld et Pascal qui, à des titres divers, mènent le même combat. L'anthropologie de cette époque - la fin notamment du règne de Louis XIV - a souvent été reconnue comme étant 'négative', et représente une réaction - 'anti-humaniste' - contre l'optimisme qui avait caractérisé les mouvements nés de la Renaissance. Après la Réforme, à l'époque de Port Royal et de Fénelon, l'on ne semble plus si sensible aux beautés et possibilités de l'être humain chantées par les générations précédentes, mais davantage au mal qui est en lui, et au danger que ce 'moi haïssable' représente pour les autres créatures.

Or, à tout cela Fénelon a une réponse radicale : ce 'moi', il faut le tuer, l'anéantir. Là où la postérité, devant les noires ambitions du 'moi', qui veut tout pour lui, proposera une dialectique avec autrui, c'est-à-dire avec les autres 'moi', pour arriver à une loi, un pacte, où les biens et les plaisirs de ce monde sont partagés raisonnablement, équitablement, Fénelon opte pour une autre perspective, purement spirituelle : le sacrifice du 'moi', réduit à son centre, à la volonté. Une telle prise de position était peut-être inévitable à l'époque où la théologie continuait à gouverner les esprits. En théologie - et pas seulement en théologie - la nouveauté commence toujours par provoquer la peur et le rejet. Il fallait sans doute passer par-là avant d'arriver à l'acceptation de ce 'moi', autorité intérieure qui, à l'époque des Lumières, allait de plus en plus prendre la place des autorités extérieures de la tradition chrétienne. Télémaque n'en annonce pas moins, par ses questions, son inquiétude et son style l'avènement de ces mêmes Lumières.

Cependant, ce qui est sacrifié ainsi, dans une perspective anthropologique, c'est le désir humain, et tout ce qui rattache le 'moi' à l'objet du désir. Le mot amour, dans Télémaque, est un concept ambigu. D'un côté, il exprime le désir amoureux, ou le désir de pouvoir qui peut en découler. Mais dans le projet spirituel dont Télémaque est la métaphore, amour renvoie à 'amour pur', c'est-à-dire au long processus d'élimination 
de tout ce qui n'est pas 'pur'. Cet amour spirituel ne prend pas sa source dans le désir, bien au contraire, il est la victoire sur le désir. De là toute l'ambiguïté qui entoure ce concept. C'est sans doute aussi la raison pour laquelle le Dieu de Fénelon, héros du pur amour, n'est pas défini en termes d'amour. Dans Télémaque la divinité est tantôt 'Loi' promulguée par Minos - tantôt 'Lumière'. Elle est surtout 'Lumière', nom qui connote une plénitude, là où l'amour évoque plutôt un vide, un 'évidement'. Mais l'image de la lumière, tout en disant la beauté de l'univers fénelonien, nous signale aussi le manque de consistance qui le menace. C'est par un jeu très subtil avec la notion de lumière que les vérités dernières sont révélées à Télémaque lors de son séjour dans le royaume de Minos. La punition des mauvais rois au Tartare est dans la 'lumière sombre' du regard propre, enfin transparent : « la vue de son propre coeur, ennemi des dieux, devient son supplice » (Fénelon $1997: 241$ ), écrit-il au sujet du roi philosophe qui n'a pensé qu'à son propre intérêt. Fénelon invente un Huis clos avant la lettre. Dans cette sombre lumière intérieure, réflexion, conscience et punition finissent par constituer une seule et même chose. Aux Champs Élysées, il nous peint la situation inverse. Ce n'est plus l'horrible auto-contemplation qui torture la créature, la divinité elle-même répand désormais sa lumière sur elle. Fénelon écrit ici peut-être les pages les plus belles et les plus inspirées de son livre :

Une lumière pure et douce se répand autour des corps de ces hommes justes, et les environne de ses rayons comme d'un vêtement. Cette lumière n'est point semblable à la lumière sombre qui éclaire les yeux des misérables mortels, et qui n'est que ténèbres ; c'est plutôt une gloire céleste qu'une lumière : elle pénètre plus subtilement les corps les plus épais, que les rayons du soleil ne pénètrent le plus pur cristal ; elle n'éblouit jamais ; au contraire, elle fortifie les yeux, et porte dans le fond de l'âme je ne sais quelle sérénité (Fénelon 1997 : 246).

Théologiquement nous reconnaissons ici le schéma augustinien, celui d'une création entièrement corrompue qui doit être aussi entièrement réparée par la grâce divine. Chez Fénelon cependant ce schéma du tout ou rien subit une transformation assez radicale. L'aversion du mauvais désir est telle qu'elle semble inspirer un refus de tout désir, même neutre ou innocent. Aussi, avec lui, 'pureté', dans le monde du désir et de l'amour, vient à signifier 'purification', et 'purification' : exclusion, victoire sur le désir donc, qui entraîne dans son rejet tout ce qui concerne ce désir. Comme objet du désir il ne reste que la volonté divine, médiatisée par la voix des Mentor de ce monde, qui doivent insuffler aux gens et à leur société le poids de vérité et de valeur auquel ils ne sauraient aspirer par eux-mêmes.

Ma conclusion peut être brève : de telles idées transposées dans le monde de la pédagogie posent de sérieux problèmes. Ainsi le désintéressement dont parle Fénelon n'aura que peu d'avenir pédagogique. Cet avenir se trouve dans la philosophie morale, chez Kant et Schopenhauer ${ }^{2}$, et dans la transformation ou plutôt la réinterprétation par les Lumières des leçons politiques et économiques du Télémaque. Il se trouve sans doute aussi dans l'attention que l'avenir ne cessera de porter à la forme de ce livre paradoxal, à son style, son art, sa saveur. De tout cela, notre colloque, à n'en pas douter, fournira de nouvelles preuves. 


\section{BIBLIOGRAPHIE}

\section{Bibliographie}

BERLAN, Françoise (1986), « Du lexique au style. Fénelon », XVII ${ }^{e}$ siècle, 231-244.

------(1995), « Lexique et affects dans le Télémaque », Littératures classiques, 9-22.

BURY, Emmanuel (1995), « La paideia du Télémaque : miroir d'un prince chrétien et lettres profanes », Littératures classiques, 69-81

------(2000), « Fénelon pédagogue », XVII ${ }^{e}$ siècle 2000, 47-56.

XVII'SIÈCLE (2000), numéro spécial Fénelon, janvier-mars, 206.

DUPRIEZ, Bernard (1961), Fénelon et la Bible, Paris, Bloud et Gay.

FÉNELON (1850), CEuvres Complètes, en 10 volumes, Paris, Gaume et autres éditeurs.

------(1983), Euvres, édition établie par Jacques Le Brun, « Bibliothèque de La Pléiade », t. I, Paris, Gallimard.

------(1997), Euvres, édition établie par Jacques Le Brun, « Bibliothèque de La Pléiade », t. II, Paris, Gallimard.

HALÉVI, Ran (dir.) (2001), Le savoir du prince, Paris, Fayard.

HEPP, Noémi (1968), Homère en France au XVII siècle, Paris, Klincksieck.

HILLENAAR, Henk (1994), Le secret de Télémaque, Paris, PUF.

KAPP, Volker (1982), Télémaque de Fénelon. La signification d'une œuvre littéraire à la fin du siècle classique, Tübingen-Paris, Gunter Narr.

LANAVÈRE, Alain (dir.) (1994), Je ne sais quoi de pur et de sublime... Télémaque, Orléans, Paradigme.

------(1995), « Les deux antiquités dans les aventures de Télémaque », Littératures classiques, 39-52.

LITTÉRATURES CLASSIQUES (1995), numéro spécial Fénelon, janvier, 23.

LE BRUN, Jacques (2001), « Du privé au public : l'éducation du prince selon Fénelon », in Halévi (dir.), 235-260.

PAPÁSOGLI, Benedetta (2000), « Espace intérieur et vie spirituelle chez Fénelon », XVII siècle, 57-72.

SPAEMANN, Robert (1963), Reflexion une Spontaneität. Studien über Fénelon, Stuttgart, Kohlhammer Verlag.

\section{NOTES}

1. Sur cette pédagogie on peut lire deux beaux articles d'Emmanuel Bury (1995 et 2000).

2. Voir l'excellente étude de Robert Spaemann (1963). 


\section{RÉSUMÉS}

Cet article étudie la signification que le Télémaque a eue pour les contemporains. Cartésien, Fénelon déplace le centre d'intérêt du projet pédagogique de 'l'objet' à enseigner vers le sujet, 'le moi' de l'élève. Le plaisir devient instrument pédagogique. D’Homère, il reprend l'histoire et le style, mais pas l'esprit joyeux, remplacé par un Christianisme austère. Sous des dehors souriants, Fénelon a une conception pessimiste de l'homme. L'élève doit être sans volonté propre, les dieux qui le gouvernent sont Minerve et Minos : sagesse sur un fond de mort. À ses yeux, le divin n'est pas 'amour' mais 'lumière'.

This article studies the meaning that Télémaque had for its contemporaries. As a Cartesian, Fénelon no longer focuses on the 'object' of teaching but on the pupil, the 'I' who is taught. Pleasure becomes a pedagogical instrument. He repeats Homer's stories and style. The joyous spirit of Ulysses is replaced by an austere Christianity. This beautiful novel conceals a pessimistic conception about mankind. The pupil has to give up his own will. Wisdom is based on the presence of death, with the reigning gods being Minerva and Minos. For Fénelon the divine is not 'love' but 'light'.

\section{INDEX}

Keywords : christian education, counselor, formation of self-concept, Mirror for Princes, pedagogy, preceptor

Mots-clés : éducation chrétienne, formation du moi, Mentor, Miroir des Princes, pédagogie, précepteur

\section{AUTEUR \\ HENK HILLENAAR}

Universiteit Groningen 